\title{
Synthesis and Bioconjugation of Gold Nanoparticles as Potential Molecular Probes for Light-Based Imaging Techniques
}

\author{
Raja Gopal Rayavarapu, ${ }^{1}$ Wilma Petersen, ${ }^{1}$ Constantin Ungureanu, ${ }^{1}$ Janine N. Post, ${ }^{2}$ \\ Ton G. van Leeuwen, ${ }^{1}$ and Srirang Manohar ${ }^{1}$ \\ ${ }^{1}$ Biophysical Engineering Group, Institute for Biomedical Technology (BMTI), Faculty of Science and Technology, \\ University of Twente, P.O. Box 217, 7500 AE Enschede, The Netherlands \\ ${ }^{2}$ Molecular Cell Biology Group, Polymer Chemistry and Biomaterials, Institute for Biomedical Technology (BMTI), \\ Faculty of Science and Technology, University of Twente, P.O. Box 217, 7500 AE Enschede, The Netherlands
}

Received 15 January 2007; Accepted 14 June 2007

Recommended by Wenxiang Cong

We have synthesized and characterized gold nanoparticles (spheres and rods) with optical extinction bands within the "optical imaging window." The intense plasmon resonant driven absorption and scattering peaks of these nanoparticles make them suitable as contrast agents for optical imaging techniques. Further, we have conjugated these gold nanoparticles to a mouse monoclonal antibody specific to HER2 overexpressing SKBR3 breast carcinoma cells. The bioconjugation protocol uses noncovalent modes of binding based on a combination of electrostatic and hydrophobic interactions of the antibody and the gold surface. We discuss various aspects of the synthesis and bioconjugation protocols and the characterization results of the functionalized nanoparticles. Some proposed applications of these potential molecular probes in the field of biomedical imaging are also discussed.

Copyright (c) 2007 Raja Gopal Rayavarapu et al. This is an open access article distributed under the Creative Commons Attribution License, which permits unrestricted use, distribution, and reproduction in any medium, provided the original work is properly cited.

\section{INTRODUCTION}

Optical imaging encompasses a multitude of techniques for the elucidation of morphology, molecular function, and metabolism of tissue with the general objective of detecting, diagnosing, staging, and treatment monitoring of disease. Progression of disease is usually accompanied by changes in physiology and pathology that are manifested as locationspecific changes in optical properties thereby providing contrast for optical imaging to study disease.

Optical imaging techniques span the range from surface to bulk imaging systems with applications ranging from "optical biopsies" to full human breast imaging with resolutions that cover the microscopic to macroscopic. Some important imaging techniques for superficial tissue imaging are confocal microscopy [1], two-photon microscopy [2], and optical coherence tomography (OCT) [3]. Techniques that permit subsurface to deep imaging are diffuse optical imaging (DOT) [4] and photoacoustic imaging [5].

The interaction of visible and near-infrared (NIR) light with tissue is dominated by (a) absorption processes which are due to the presence of various chromophores such as hemoglobin, oxyhemoglobin, melanin, water, and lipids [6];

(b) scattering processes due to the cell membrane and cell structures such as the nucleus, mitochondria, lysosomes [6].

Penetration of light in tissue is dependent on the extent of the two processes above and is low in the high-energy visible region of the spectrum. This is due to high absorption by hemoglobin and severe light scattering. In the wavelength regime between $600 \mathrm{~nm}$ and $1100 \mathrm{~nm}$, absorption and scattering losses are minimal permitting high-light penetration. This is the so-called "optical imaging window" which is exploited for deep imaging in tissue [7].

The sensitivity and specificity of optical imaging techniques to visualize a pathological disorder are governed by contrast: the ability of the disease to differentially scatter or absorb light compared with nonpathological tissue and background noise. This native or endogenous contrast may not be sufficient and in any case, the interactions of light with tissue 
are not disease-specific. Therefore, there is a role for exogenously administered contrast enhancing agents which have affinity for the disease site through biochemical interactions, providing not only sensitive but also disease-specific signals.

Contrast agents for optical imaging thus far have nearinfrared dyes based on cyanine dyes [8] such as Indocyanine Green [9], but in the last few years, gold nanoparticles [10 12] have emerged as prime candidates due to their unusual optical properties and inherent biocompatibility.

Gold metal nanoparticles (NPs) exhibit narrow and intense absorption and scattering bands due to the phenomenon of plasmon resonance. This occurs at the resonance condition of the collective oscillation that the conduction electrons experience in an electromagnetic field of the appropriate wavelength [13]. The plasmon resonant condition of gold NPs depends upon their size, shape, structure (solid or hollow), and upon the refractive index of the embedding medium. Spherical gold nanoparticles have a single plasmon resonant extinction peak at around $520 \mathrm{~nm}$, which does not shift extensively with changes in size and refractiveindex of the surrounding medium. This is a wavelength at which light penetration in tissue is poor due to strong scattering and absorption by hemoglobin, and gold nanospheres are not useful in contrast enhancement for deep tissue imaging.

Rod-shaped NPs exhibit two plasmon resonances due to oscillation of the conduction electrons along the short axis as well as along the long axis of the particles. The former plasmon band is called the transverse resonance and the latter the longitudinal resonance. While the transverse plasmon band occurs in the neighborhood of $520 \mathrm{~nm}$, the longitudinal band is red-shifted. The extent of the red-shift depends on the aspect ratio of the nanorod; the higher the aspect ratio, the further the shift. Thus by tailoring the length and/or width of these particles, their extinction peaks may be made to cover the low-energy visible to infrared wavelength regions.

The intense scattering and absorption of light, that occurs under the plasmon resonant condition coupled with the ability to tune the resonance into the near-infrared (NIR) by manipulating the aspect ratio, make gold nanorods extremely attractive as contrast agents for optical imaging techniques. Further, gold-protein chemistry is well developed and several bioconjugation protocols are available in the literature, which allows the combination of the targeting functionality of antibodies with such gold NPs. The inertness and biocompatibility of gold in general hold promise the use of gold NPs for in vivo imaging applications.

Gold NPs can be synthesized using wet chemical methods which are based on the reduction of gold salts by reagents such as sodium borohydride and ascorbic acid. Seed-mediated methods dominate wet chemical synthesis routes. These involve the reduction of gold using weak reducing agents onto small nanospheres of gold as seed, in the presence of shape-directing surfactants usually cetyl trimethylammonium bromide (CTAB). These methods may be distinguished into those that use silver ion assistance in growth solutions and those that do not.

Murphy and coworkers described the three-step growth protocol $[14,15]$, where medium to high aspect ratio nanorods could be synthesized, without the use of silver nitrate. Seed particles are generated by reducing gold salt using sodium borohydride in the presence of sodium citrate. The spheres are coated with a layer of negatively charged citrate ions that maintain colloid stability against aggregation by electrostatic repulsion. These spheres seed a growth solution comprising gold salt, CTAB, and ascorbic acid in three steps thereby slowing down reduction. The mechanism of nanorod formation by this method is not yet fully understood. Murphy et al. [15] proposed that the polar $\mathrm{CTA}^{+}$ head group of the surfactant binds with greater preference to certain crystallographic faces thereby passivating them to the deposition of gold. The other faces, on the other hand, would be exposed for gold to be reduced on, thereby producing anisotropic growth into rods.

The methods using silver nitrate in the growth solutions were proposed by Jana et al. [16], but modified by Nikoobakht and El-Sayed [17] to achieve spectacular yields of nanorods with excellent monodispersity. Importantly, they also showed that changing the quantity of $\mathrm{Ag}^{+}$ions in the growth solution allows for fine-tuning of the aspect ratios of the nanorods. The mechanism at work in this protocol has been debated in the recent past. One mechanism postulates CTAB as a soft template which elongates on addition of $\mathrm{Ag}^{+}$ ions which occupy regions between the $\mathrm{CTA}^{+}$head groups to reduce the repulsion between the head groups [17]. A second mechanism invokes the CTAB passivation concept with additional adsorption of silver bromide on facets slowing down reduction and producing rods shorter than those made without using $\mathrm{Ag}^{+}$[18]. A third mechanism which has appeared recently [19] proposes the underpotential deposition of $\mathrm{Ag}^{0}$ on certain faces, followed by CTAB binding, which serves to stabilize the faces, and allows gold reduction on other faces resulting in rod formation.

In this article, we present our experiences in synthesizing gold nanospheres and nanorods using slight modifications of the protocols discussed above. Our goal is to obtain nanorods whose aspect ratios can be tuned to obtain plasmon peaks between $650 \mathrm{~nm}-850 \mathrm{~nm}$. Next, we conjugate the gold nanospheres and gold nanorods to the HER 81 monoclonal antibody using electrostatic and hydrophobic interactions. The conjugation does not use modifications of the bilayer charge of nanorods nor does it use any linkers. We discuss various aspects of these protocols and postulate a possible mechanism for the bioconjugation of the antibody with the gold nanorods. We also discuss the feasibility of using these molecular probes for contrast enhancement of photoacoustic cancer imaging using simulations.

\section{EXPERIMENTAL SECTION: MATERIALS AND METHODS}

\subsection{Gold nanorods using the silver-assisted single surfactant growth method}

As mentioned earlier, the seed-mediated protocol requires the use of small gold nanospheres to seed growth solutions with silver nitrate as per the protocol of Nikoobakht and ElSayed [17]. 
TABLE 1: Mean aspect ratios, lengths, widths, and longitudinal plasmon peaks for nanorods synthesized using the silver-assisted growth method.

\begin{tabular}{cccccc}
\hline Sample & Volume of $\mathrm{AgNO}_{3}(\mu \mathrm{L})$ & Aspect ratio $(\mathrm{R})$ & Length $(\mathrm{nm})$ & Width $(\mathrm{nm})$ & LP band $(\mathrm{nm})$ \\
\hline 1 & 50 & $2.3 \pm 0.3$ & $44.8 \pm 4.1$ & $19.8 \pm 2.9$ & 675 \\
2 & 100 & $2.85 \pm 0.6$ & $45.1 \pm 5.5$ & $15.8 \pm 3.1$ & 764 \\
3 & 150 & $3.0 \pm 0.6$ & $41.7 \pm 3.9$ & $13.9 \pm 2.3$ & 788 \\
4 & 200 & $3.1 \pm 0.5$ & $52.0 \pm 4.6$ & $16.8 \pm 2.8$ & 831 \\
5 & 250 & $3.6 \pm 0.6$ & $51.0 \pm 4.4$ & $14.1 \pm 2.1$ & 850 \\
\hline
\end{tabular}

The following are the reagents used for the synthesis of the gold seed and gold nanorods.

Tetrachloroauric acid $\left(\mathrm{HAuCl}_{4} \cdot 3 \mathrm{H}_{2} \mathrm{O}\right)$ was purchased from Acros Organics (Belgium), hexadecyltrimethylammonium bromide (CTAB > 99\%), sodium borohydride $\left(\mathrm{NaBH}_{4}, 99 \%\right)$, and ascorbic acid (99\%) from Aldrich (The Netherlands) and silver nitrate $\left(\mathrm{AgNO}_{3}, 99.8 \%\right)$ from Merck (Germany). Prior to use, all glassware was cleaned with hydrofluoric acid ( $\mathrm{HF})$, further with aqua regia $\left(\mathrm{HCl} / \mathrm{HNO}_{3}\right)$ and rinsed twice with deionized water.

\section{Gold seed of $3.5 \mathrm{~nm}$ diameter}

The synthesis was done using protocols of Nikoobakht and El-Sayed [17] with slight modifications. A solution of CTAB $(5 \mathrm{~mL} ; 0.2 \mathrm{M})$ was sonicated for 20 minutes at $40^{\circ} \mathrm{C}$ in a water bath. A solution of $\mathrm{HAuCl}_{4} \cdot 3 \mathrm{H}_{2} \mathrm{O}(5 \mathrm{~mL} ; 0.0005 \mathrm{M})$ was added with continuous stirring under inert conditions (nitrogen environment). Then, an ice-cold aqueous solution of $\mathrm{NaBH}_{4}(0.6 \mathrm{~mL} ; 0.01 \mathrm{M})$ was added at once with vigorous stirring for 1 minute. This seed solution (CTAB-capped) is further used during growth stage of nanorods.

\section{Gold nanorods of varying aspect ratios}

Five identical conical flasks containing $10 \mathrm{~mL}$ of a growth solution that consists of CTAB $(5 \mathrm{~mL} ; 0.2 \mathrm{M})$ and $\mathrm{HAuCl}_{4}$. $3 \mathrm{H}_{2} \mathrm{O}(5 \mathrm{~mL} ; 0.001 \mathrm{M})$ were prepared. The color of the growth solution is dark-yellow. $\mathrm{AgNO}_{3}(50,100,150,200$, and $250 \mu \mathrm{L}$ of $0.006 \mathrm{M}$ ) was added to the five identical growth solution flasks in an amount that was chosen so as to yield desired aspect ratios for the resulting nanorods. Following this, the mild reducing agent ascorbic acid $(70 \mu \mathrm{L} ; 0.1 \mathrm{M})$ was added to each growth solution conical flask to give colorless solutions. Finally, $14 \mu \mathrm{L}$ of preformed CTAB-capped seed solution was added to each conical flask, and mixtures were gently mixed. After 3 hours at $24^{\circ} \mathrm{C}$, the nanorod suspension turned into a dark-blue solution with a brownish opalescence. These solutions were concentrated by centrifuging twice at $12000 \mathrm{~g}$ for 20 minutes which also enabled removal of the excess unbound CTAB. The centrifuged gold nanorods which are dispersed in water were stored at $4^{\circ} \mathrm{C}$.

\subsection{Characterization of gold nanoparticles}

Electron microscopy of the NPs was performed using a CM 30 Philips transmission electron microscope (TEM) or a Zeiss-1550 high-resolution scanning electron microscope
(HRSEM). Particle sizes were estimated using NI Vision module (Labview, National Instruments) on the digital SEM images with at least 250 particles considered in each case. Extinction spectra of NPs (and bioconjugated NPs) were measured using the Shimadzu PC3101 UV-Vis-NIR spectrophotometer.

The concentration of nanorods synthesized was estimated using the relation $A=c d \varepsilon$, where $A$ is the measured absorbance, $c$ the concentration in moles $(\mathrm{M}), \varepsilon$ the molar extinction coefficient $\left(\mathrm{M}^{-1} \mathrm{~cm}^{-1}\right)$, and $d$ the path length of the cuvette used to record the spectra. The derived molar extinction coefficients can be compared with $\varepsilon$ values from a recent report, estimated for a range of aspect ratios of nanorods by measuring the gold content in sols using inductively coupled plasma (ICP) atomic emission spectroscopy [19].

\subsection{Bioconjugation of HER81 $\mathrm{mAb}$ to gold nanoparticles}

Conjugation was achieved using combination of electrostatic and hydrophobic binding interactions. The particles chosen for bioconjugation were $25 \mathrm{~nm}$ citrate-capped gold spheres (Aurion, Wageningen, The Netherlands), and silver-assisted surfactant mediated gold nanorods with aspect ratios of approximately 2.85 (see Table 1 ) with the longitudinal plasmon peak at $764 \mathrm{~nm}$. The anti-HER 2 mouse monoclonal antibody (mAb) (Immunicon, USA) was chosen as the targeting moiety. The antibody designated as HER81 recognizes Human EGF receptor 2, HER2. HER2, also called erbB2, is a member of the epidermal growth factor receptor (EGFR) family and is overexpressed in $20-40 \%$ of human breast cancers [20].

In general, for optimum conjugation, it is recommended that the $\mathrm{pH}$ of the antibody and gold sol be maintained at or slightly higher than the isoelectric point (pI) of the antibody [21]. The isoelectric (pI) point of HER81 mAb was determined using the Pharmacia PhastSystem isoelectric focusing (IEF). The $\mathrm{pH}$ of the antibody was adjusted with dialysis in $5 \mathrm{mM}$ sodium acetate buffer and the $\mathrm{pH}$ of the colloidal gold was adjusted with $0.1 \mathrm{M} \mathrm{KOH}$, to approximately $0.5 \mathrm{pH}$ units above this value.

Next, the minimum protecting amount of antibody to be used for the conjugation is determined. This is the amount of protein that is required to maintain colloidal stability of the conjugated NPs upon addition of $\mathrm{NaCl}$ [21] as judged by colorimetric analysis; as long as the conjugated NPs turns blue, particle aggregation takes place implying that the amount of protein is not sufficient to stabilize the suspension. By trial, different amounts of antibody are added to samples of the 


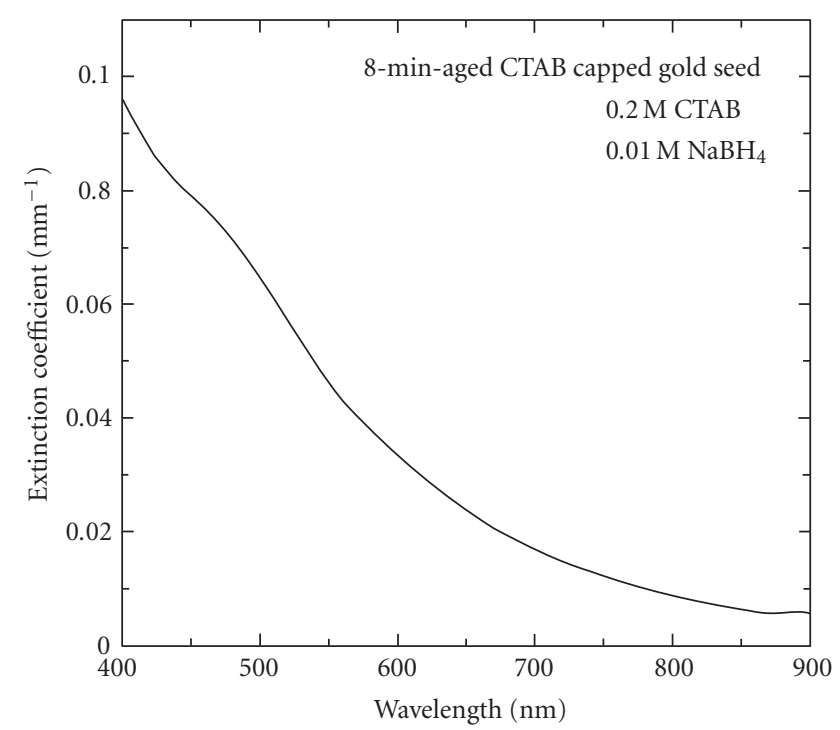

FIgURE 1: Optical extinction spectrum of preformed 8-minute-aged CTAB-capped gold nanospheres as seed for nanorod synthesis.

gold sol, gently mixed, and allowed to stand at room temperature for 2 minutes. Spectroscopic analysis reveals which sample remains stable; the minimum amount of protein added is then ascertained and is used for subsequent conjugation of the gold sol.

To block the free surfaces on the gold, 10\% bovine serum albumin (BSA) in dialyzed buffer (sodium acetate), maintained at the same $\mathrm{pH}$ as the antibody solution, is used. The BSA was added to the conjugate to a final concentration of $1 \%$ and was allowed to incubate for 5 minutes. The resultant was then centrifuged for 30 minutes at $12000 \mathrm{~g}$ to remove excess protein and incompletely stabilized particles. The resulting pellet is dispersed in phosphate buffered saline (PBS) containing $1 \%$ BSA and stored at $4^{\circ} \mathrm{C}$.

\subsection{Cell culture and cell-bioconjugate incubation}

The HER2 positive mammary adenocarcinoma (SKBR3) cell line was used as a positive cell line; Chinese hamster ovary ( $\mathrm{CHO}$ ) cells were used as an HER2 negative cell line. The cells were cultured in RPMI 1640 medium (Invitrogen) supplemented with glutamine, 10\% FBS (Fetal Bovine Serum) with antibiotics. Cells were maintained in an incubator at $37^{\circ} \mathrm{C}$ and $5 \% \mathrm{CO}_{2}$.

The cells were detached from the tissue culture plate using trypsin. The cells were replated onto $12 \mathrm{~mm}$ glass cover slips in a 6-well tissue culture plate, and allowed to grow for 2 days at $37^{\circ} \mathrm{C}, 5 \% \mathrm{CO}_{2}$. When the cells grew to $80 \%$ confluence on the cover slips, the cells were rinsed with phosphate buffered saline (PBS) and fixed in 4\% paraformaldehyde (PFA) for 15 minutes at room temperature.

\subsection{Immunostaining and confocal microscopy}

After fixation, immunostaining was performed on the cells. The cells were incubated with $100 \mu \mathrm{L}$ of conjugated gold nanoparticles at a concentration of $9.7 \times 10^{10}$ particles per $\mathrm{mL}$ for 2 hours followed by silver enhancement performed using a silver-staining kit (Aurion, Wageningen, The Netherlands).

Confocal microscopy reflection images of the cells on cover slips were recorded on a Zeiss LSM 510 confocal laser scanning microscope using a C-Apochromat $63 \mathrm{X} / 1.4 \mathrm{nu}-$ merical aperture (NA) water-immersion objective. An excitation wavelength of $543 \mathrm{~nm}$ was chosen and reflection images recorded using a 500-550 nm bandpass filter. All images were acquired with pinhole diameters of $178 \mu \mathrm{m}$. Care was taken to ensure that the excitation intensity as well as detector and amplifier gains were maintained at the same values for all images to facilitate comparison.

\section{RESULTS}

\subsection{Synthesis of gold nanorods}

We used seed particles within about 8 minutes of formation in the subsequent growth phase. The optical extinction spectrum of 8 -minute-aged seed is shown in Figure 1.

Figure 2 shows the extinction spectrum and HR-SEM image of the nanorods synthesized using $50 \mu \mathrm{L} \mathrm{AgNO}_{3}$ in the growth solution. The peak at $675 \mathrm{~nm}$ can be attributed to longitudinal plasmon resonance and the peak in the vicinity of $516.5 \mathrm{~nm}$ to transverse plasmon resonance.

Examination of the SEM image and determination of the mean sizes confirm that the NPs produced consist of monodisperse nanorods of aspect ratio of $2.3 \pm 0.3$, with a small number of large spheres; the latter's extinction peak coinciding with the transverse plasmon band of the nanorods. Figure 3 shows the extinction spectrum and the SEM image for the sample produced using $250 \mu \mathrm{L}$ of silver nitrate. It is seen that the longitudinal plasmon band is shifted to $850 \mathrm{~nm}$. Sizing from the SEM image yields an average aspect ratio of $3.6 \pm 0.6$. Figure 4 shows the size distribution of the 2 specimens.

The values of the molar extinction coefficient for the 2 cases above are $3.3 \pm 0.3 \times 10^{9}$ and $5.5 \pm 0.3 \times 10^{9} \mathrm{M}^{-1} \mathrm{~cm}^{-1} \mathrm{ob}-$ tained by extrapolation of the data as reported in [19]. With this, we arrive at the concentration of the nanorods with peak at $675 \mathrm{~nm}$ as $4.3 \pm 0.3 \times 10^{11} \mathrm{NR} / \mathrm{mL}$; for nanorods with the peak at $850 \mathrm{~nm}$ as $1.3 \pm 0.7 \times 10^{11} \mathrm{NR} / \mathrm{mL}$.

Figure 5 shows the consolidated normalized extinction spectra of 5 nanorod solutions, having identical growth solutions with varying silver nitrate volumes. The spectra were normalized to the peak at $516 \mathrm{~nm}$, which is due to a combination of the transverse plasmon resonance of the nanorods and the signature peak of gold nanospheres. It is seen that with higher silver nitrate volumes the extent of red-shifting increases [22]. The details of the observed changes in aspect ratios and plasmon bands are presented in Table 1.

\subsection{Bioconjugation of gold nanospheres and gold nanorods}

A signature for successful binding of protein to gold NPs is a red-shifted and amplitude reduced plasmon band. Both 


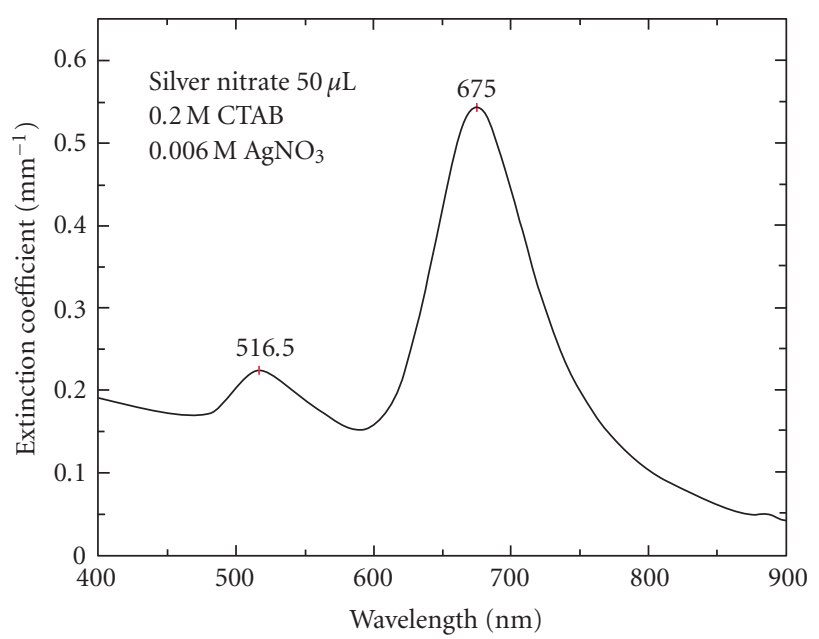

(a)

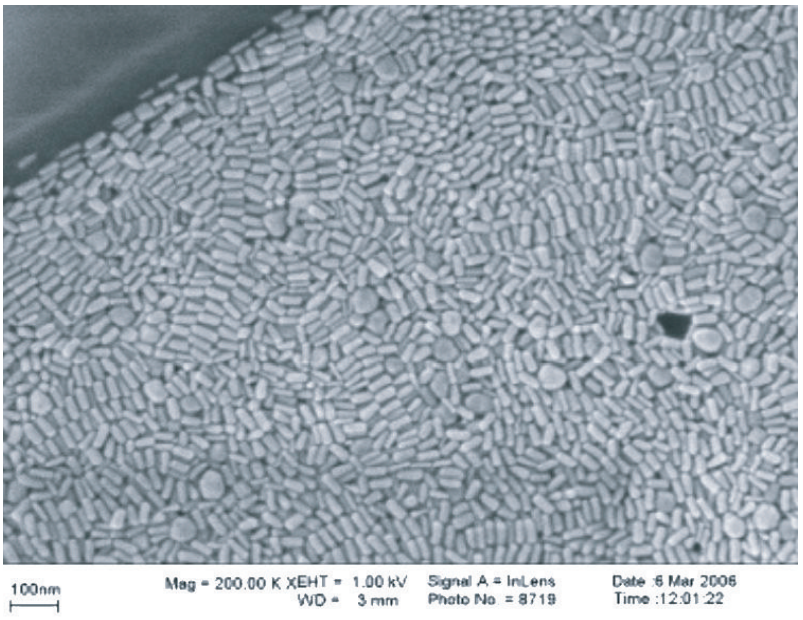

(b)

FIGURE 2: Gold nanorods synthesized using $50 \mu \mathrm{L}$ of silver nitrate in growth solution. (a) Optical extinction spectrum showing the transverse plasmon peak at $516.5 \mathrm{~nm}$ and the longitudinal plasmon peak at $675 \mathrm{~nm}$. The amplitude of the longitudinal plasmon peak is higher than transverse plasmon peak which indicates the formation of high yield of nanorods compared to spheres. (b) High-resolution scanning electron microscope (SEM) image of gold nanorods showing high monodispersity. Few nanospheres are observed.

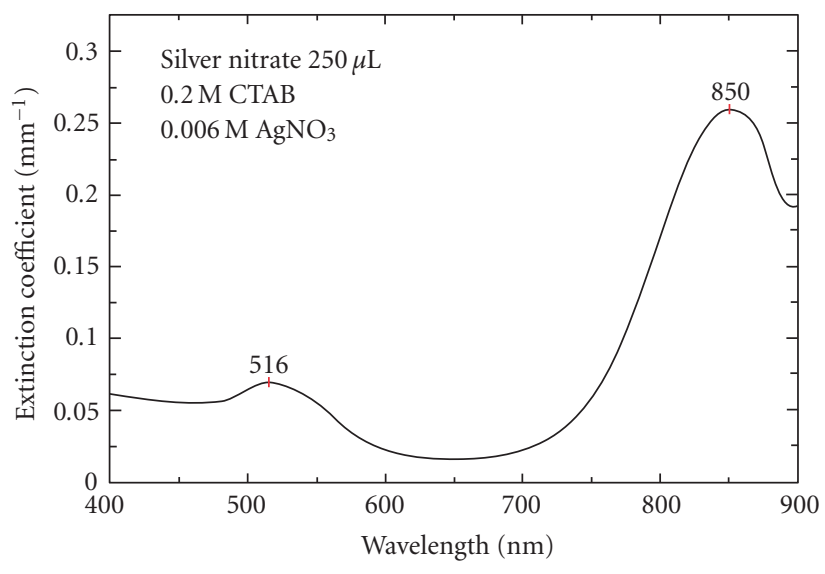

(a)

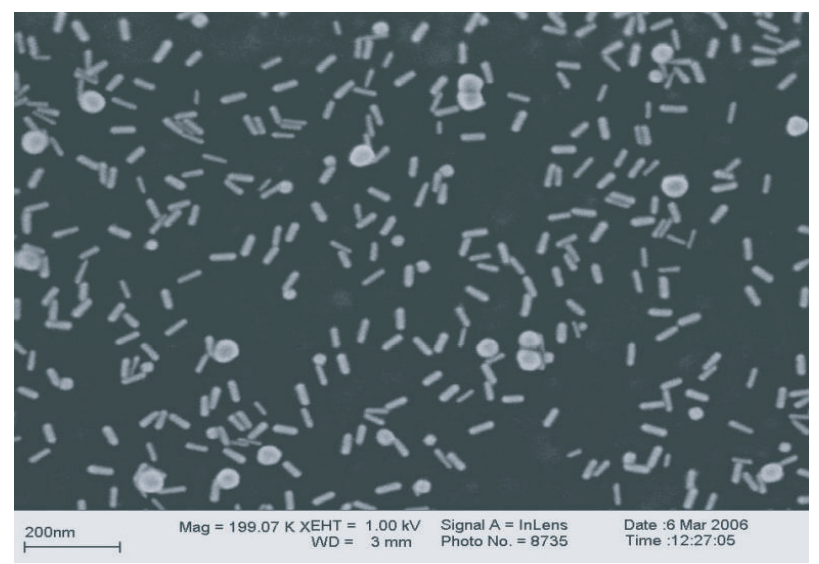

(b)

FIGURE 3: Gold nanorods synthesized using $250 \mu \mathrm{L}$ of silver nitrate in growth solution. (a) Optical extinction spectrum showing the transverse plasmon peak at $516 \mathrm{~nm}$ and the longitudinal plasmon peak at $850 \mathrm{~nm}$. (b) High-resolution scanning electron microscope (SEM) image.

these effects are due to the formation of the inhomogeneous layer of protein on the gold particle surface that leads to the modification of refractive index of the embedding medium.

Figure 6(a) shows the extinction spectrum of gold nanospheres before and after incubation with HER81. Figure 6(b) is the corresponding situation with gold nanorods before and after incubation with the HER81 mAb. In both cases, the characteristic red-shift in the extinction peak of the plasmon bands is seen.

Not too much should be read into the amplitude changes of the extinction spectra since centrifugation of the bioconjugate to remove unbound protein, redispersion in water, and other procedures results in a change in the concentration of the NPs used for spectroscopy.
Figure 7(a) show confocal reflectance image (on left) and bright field image (on right) of the HER $81 \mathrm{mAb} /$ gold sphere conjugates incubated with SKBR3 cells. As discussed in the experimental section, silver enhancement was used by which silver is reduced onto the gold particles forming large clusters around $500 \mathrm{~nm}$ in size. This then enables visualization under the microscope. The HER2 receptors are localized to the cell membranes of SKBR3 cells. The high intensities in both images at the cell membrane are then evidence of the preservation of the functionality of the antibody and also illustrate successful conjugation. The images in Figure 7(b), which show the situation with the negative control using the $\mathrm{CHO}$ cells, display no such accumulation of gold particles. Also, adding nonantibody conjugated gold NPs to the SKBR3 cells did not result in accumulation of the nanorods, indicat- 


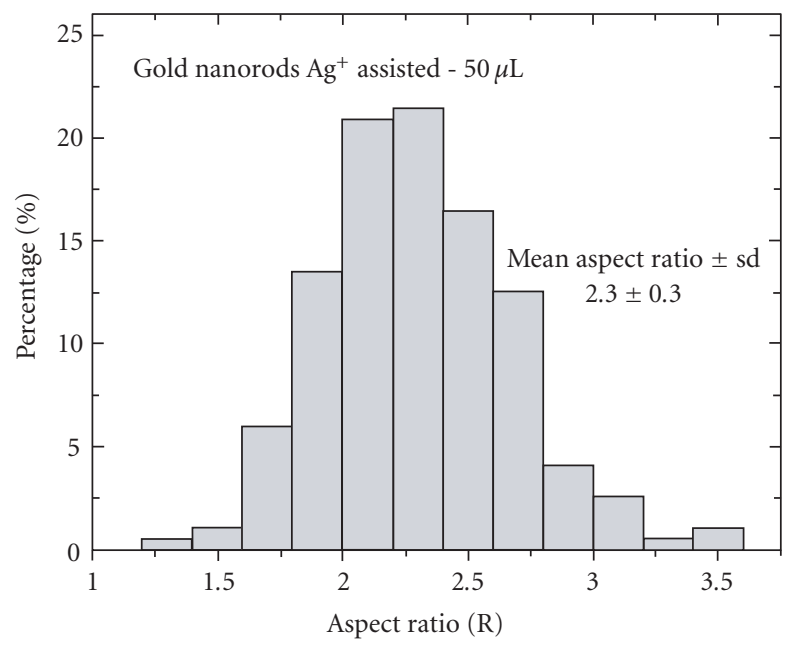

(a)

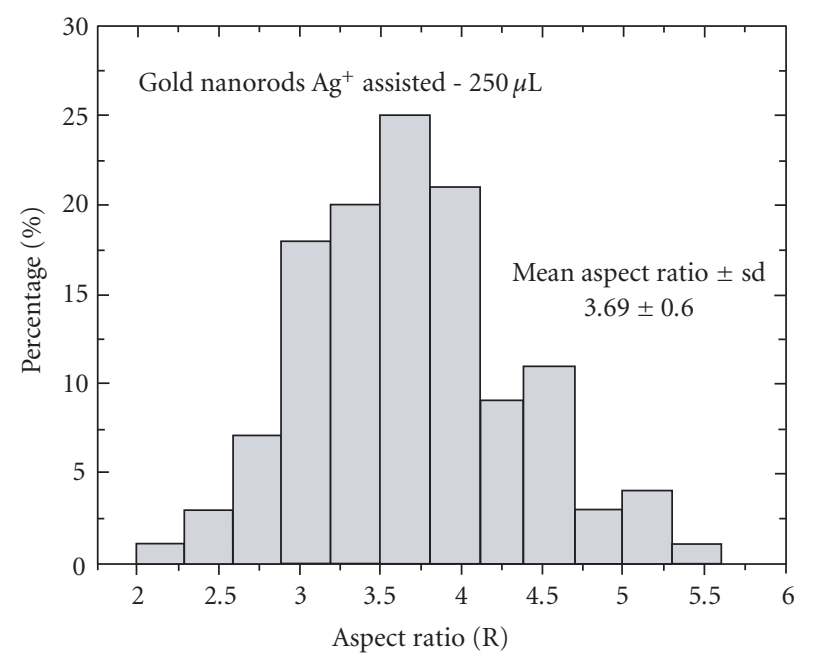

(b)

FIGURE 4: Histograms of gold nanorod aspect ratios synthesized with (a) $50 \mu \mathrm{L}$ silver nitrate, mean aspect ratio of $2.3 \pm 0.3$ (mean length $44.8 \pm 4.1 \mathrm{~nm}$, mean width $19.8 \pm 2.9 \mathrm{~nm}$ ); and with (b) $250 \mu \mathrm{L}$ silver nitrate, mean aspect ratio of $3.6 \pm 0.6$ (mean length $51.0 \pm 4.4 \mathrm{~nm}$, mean width $14.1 \pm 2.1$ ).

ing the specificity of the HER81 antibody-nanorod conjugate (data not shown).

Figures $8(\mathrm{a})$ and $8(\mathrm{~b})$ are the results of the corresponding controls using the HER81 mAb/gold nanorods.

\section{DISCUSSION}

\subsection{Gold nanorod synthesis}

The end products of the seed-mediated growth protocols are crucially dependent on the nature of the seed, upon their size and upon the capping agents used. Additionally, the constituents and their concentrations in the growth solution influence the outcome of the synthesis products. The addition of silver ions in the growth solution and the use of preformed CTAB stabilized seed in the protocol of Nikoobakht and El-Sayed [17] produced not only a high yield of monodisperse nanorods but fine tunability of aspect ratios.

There are many unanswered questions regarding the mechanism of formation of gold nanorods using the silverassisted protocol and this has been the topic of several studies [15, 17-19]. Recent reports of Orendorff and Murphy [19], and Liu and Guyot-Sionnest [23] provide some insights into the mechanisms that could be involved in the synthesis. It is postulated that silver ions are reduced by ascorbic acid even though it is a weak reducing agent, by the phenomenon of underpotential deposition (UPD). This is reduction of silver in monolayers on the growing gold nanorod surface at a potential less than the standard reduction potential [19]. The deposition is not uniform on the gold surface but occurs faster on the sidewalls compared with the end faces. Remarkably, the sidewalls in the case of nanorods produced with silver assistance using $\mathrm{CTAB}$ protected seed bear $\mathrm{Au}\{110\}$ faces, while the end faces have $\mathrm{Au}\{100\}$ faces. This is in contrast to the rods prepared by using citrate-capped seed without $\mathrm{Ag}^{+}$. This faster passivation of the sidewalls is followed by CTAB

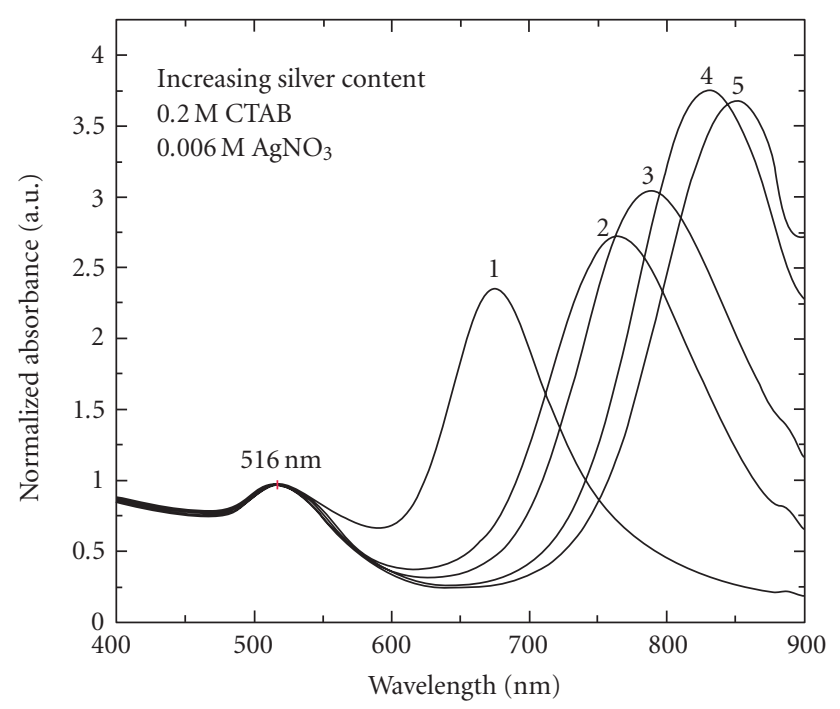

FIGURE 5: Normalized extinction spectra of gold nanorods with increasingly red-shifted longitudinal plasmon bands, synthesized using $50,100,150,200$, and $250 \mu \mathrm{L}$ of silver nitrate in the growth solution for curves $1-5$, respectively. Normalization of the spectra is done with respect to the transverse plasmon peak amplitudes.

binding possibly via bromide ions. This inhibits the reduction of gold, which deposits on the end faces. Ultimately, the end faces are also stabilized preventing the formation of very long nanorods. The model claims also to explain the increase in aspect ratio of the nanorods produced with higher concentration of silver ions used, by proposing that higher UPD of silver monolayers occur on the sidewalls which one assumes reducing the width of the nanorods thus increasing the aspect ratios [19].

Indeed, we observe some phenomena that are consistent with the above model. We are able to synthesize gold 


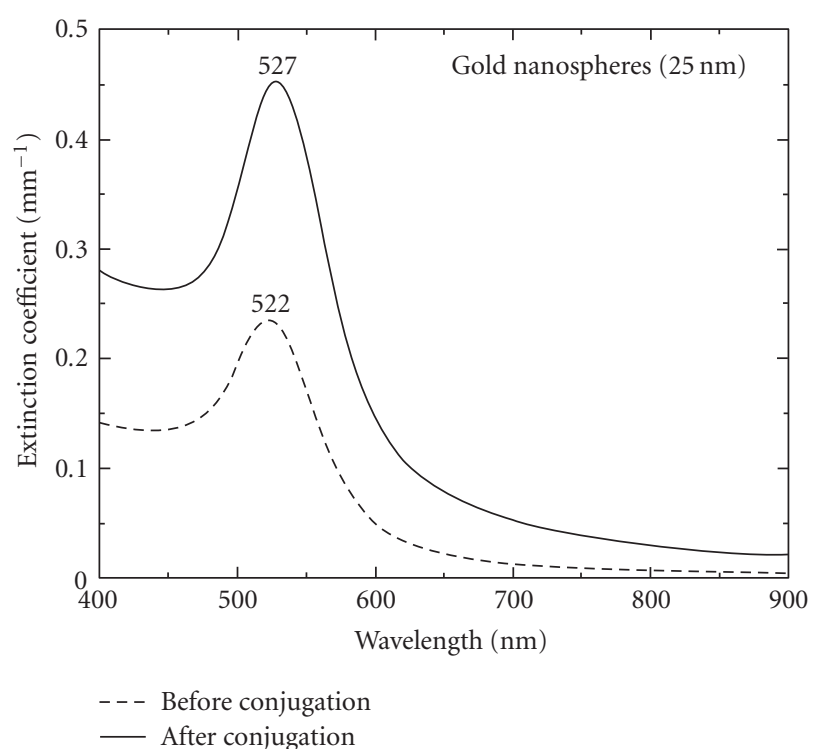

(a)

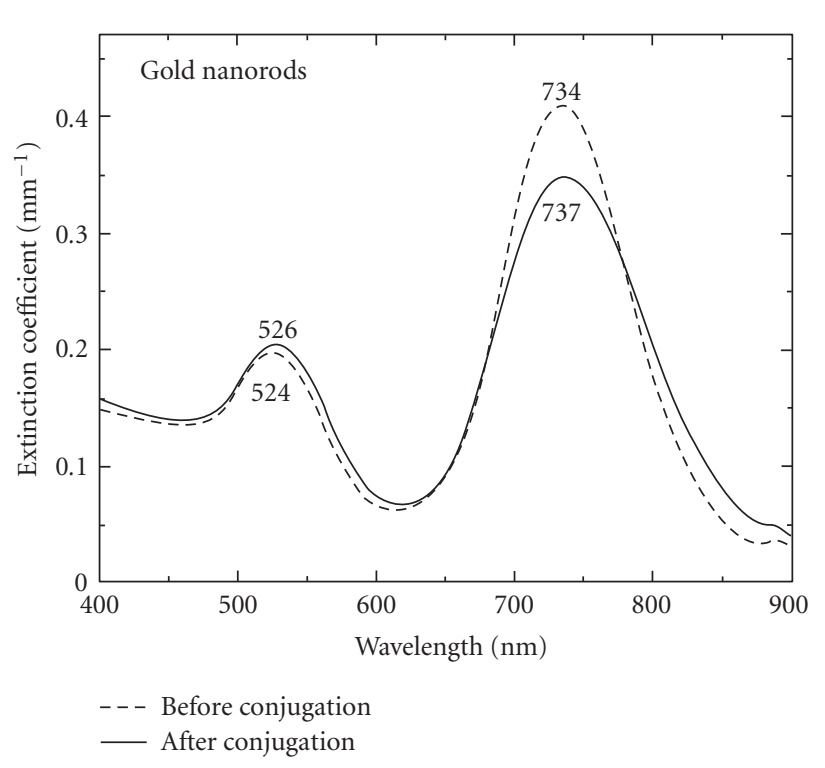

(b)

FIGURE 6: Extinction spectra before and after incubation of HER81 with (a) gold nanospheres, (b) gold nanorods. In both cases, a red shift in plasmon band(s) occurs after incubation with the antibody signifying successful bioconjugation.

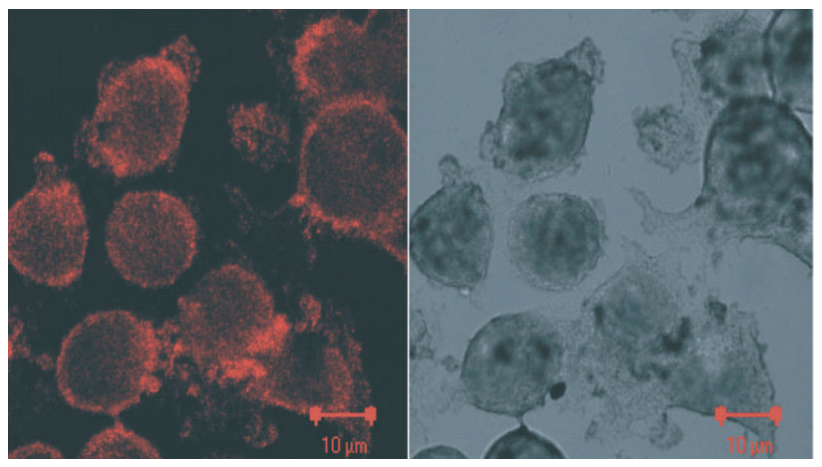

(a)

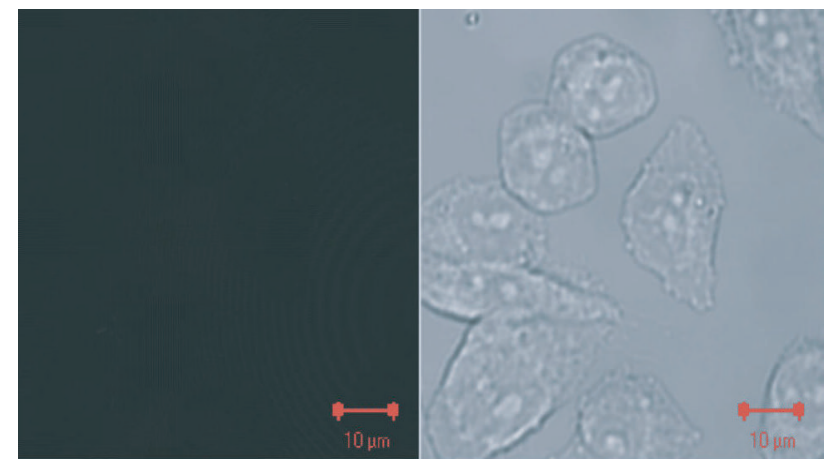

(b)

FIGURE 7: Confocal reflectance images (left) and bright field images (right) of (a) SKBR3 cells incubated with silver-stained HER81/gold sphere conjugates, (b) CHO cells under the same conditions. Care was taken to maintain the same acquisition parameters in both cases. The silver-stained bioconjugates are detected at the cell membranes of SKBR3 cells where HER2 is localized. This indicates successful conjugation and retention of functionality of the antibody after conjugation. No such accumulation of HER81/gold sphere conjugates is demonstrated in HER2 negative $\mathrm{CHO}$ cells.

nanorods only up to an aspect ratio of 3.6, as shown in Figure 3. Addition of higher volumes of silver nitrate produces no further increase in the aspect ratios of the particles. These particles have an average length of $51 \mathrm{~nm}$. This supports the idea that ultimately complete passivation of the entire nanorod surface occurs preventing further gold deposition even though the reagents have not been exhausted. Further, we observe that nanorods that are made with increasing $\mathrm{Ag}^{+}$volumes have smaller diameters with the lengths practically unchanged or only slightly increasing (see Table 1 ). The above model can also explain this. It must be mentioned that the model does not have an appealing explanation regarding the ability to tune the aspect ratios so precisely by $\mathrm{Ag}^{+}$vari- ation. It is very likely that the model will have to undergo refinements or even major changes before it is universally accepted.

\subsection{Gold nanoparticle-antibody conjugation}

The noncovalent conjugation of proteins to colloidal gold is usually due to a combination of electrostatic and hydrophobic interactions. Citrate-capped gold NPs are negatively charged due to a layer of negative citrate ions. Positively charged amino groups of the antibody will be attracted to the gold surface, and when the protein comes close enough for binding, the hydrophobic pockets of the protein will make contact and bind with the gold [24]. A general guideline to 


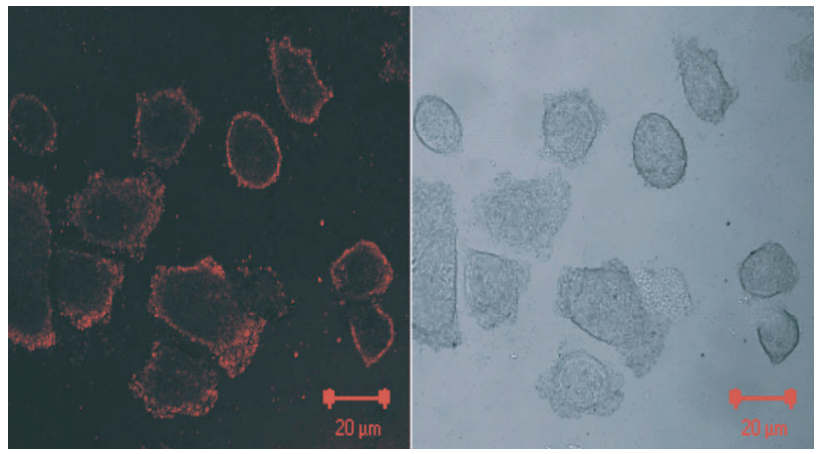

(a)

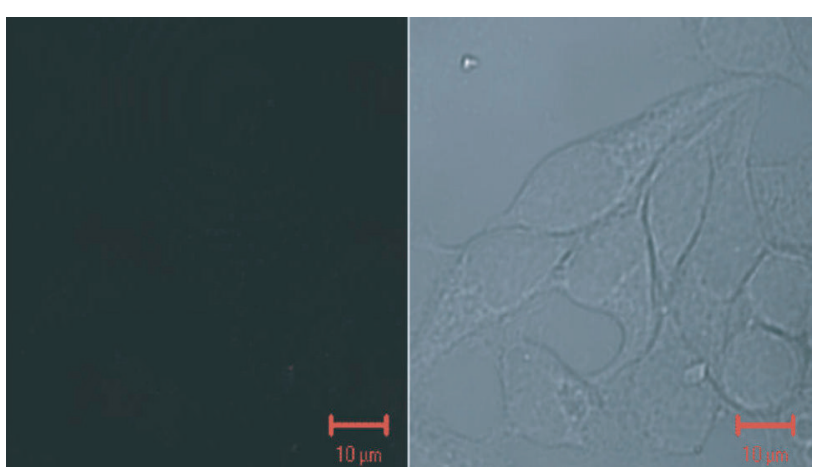

(b)

FIGURE 8: Corresponding images as in Figure 7 for bioconjugates consisting of silver-stained HER81/gold nanorod conjugates incubated with (a) SKBR3 cells and (b) CHO cells. Care was taken to maintain the same acquisition parameters in both cases. The bioconjugates are accumulated at the cell membranes of SKBR3 cells where HER2 is localized. As expected, no such accumulation takes place in the case of HER2 negative CHO cells.

TABLE 2: Important optical imaging techniques that utilize absorption and scattering contrasts in biology and medicine.

\begin{tabular}{|c|c|c|c|c|}
\hline Technique & Imaging depth & Imaging resolution & Mechanism & Typical imaging applications \\
\hline Confocal microscopy [1] & $500 \mu \mathrm{m}$ & $>250 \mathrm{~nm}$ & Scattering /absorption & Tissue surfaces \\
\hline Two-photon microscopy [2] & $800 \mu \mathrm{m}$ & $>250 \mathrm{~nm}$ & Absorption & Tissue surfaces \\
\hline $\begin{array}{l}\text { Optical coherence } \\
\text { tomography [3] }\end{array}$ & $2 \mathrm{~mm}$ & $1 \mu \mathrm{m}$ & Scattering & $\begin{array}{l}\text { Surfaces/subsurfaces } \\
\text { of tissue }\end{array}$ \\
\hline Diffuse optical tomography [4] & $>20 \mathrm{~mm}$ & $\approx 10 \%$ depth & Scattering/absorption & $\begin{array}{l}\text { Small animal; } \\
\text { human breast and brain }\end{array}$ \\
\hline Photoacoustic imaging [5] & $>20 \mathrm{~mm}$ & $\begin{array}{l}<1 \mathrm{~mm} \\
\text { (detector bandwidth limited) }\end{array}$ & Absorption & $\begin{array}{l}\text { Subsurface to deep imaging; } \\
\text { small animal; human breast }\end{array}$ \\
\hline
\end{tabular}

optimize the bioconjugation is that the $\mathrm{pH}$ of the antibody and the gold sol must be maintained at or slightly above the isoelectric point of the antibody [24]. Following this, procedure for citrate-capped gold nanospheres with the HER81 antibody resulted in good bioconjugation as shown by the spectroscopy and bioactivity studies (Figure 7).

With nanorods, the situation is more complex as compared with nanospheres. The sidewalls of the nanorods are expected to be stabilized with a bilayer of CTAB, which imparts a positive charge to the gold. Huang et al. [25] first changed the positively charged surface to a negatively charged one by exposing the nanorods to poly(styrenesulfonate) PSS polyelectrolyte solution. The PSS-capped nanorods were then treated in the same way as in Section 2.3 with the conjugation being done with anti-EGFR monoclonal antibodies.

Zeta potentials of the gold nanorod solution as originally prepared were determined to be $+55 \mathrm{mV}$. Centrifugation to remove the excess unbound $\mathrm{CTAB}$ and redispersion of the rods in water saw a reduction in the zeta potential to $+7.5 \mathrm{mV}$, which also points to a low stability. We surmise that inspite of the net positive charge, the unpassivated end faces would be negatively charged due to the presence of $\mathrm{AuCl}^{-2}$ ions [21]. We therefore performed the same protocol (as in Section 2.3) and found that the bioconjugation indeed was achieved as demonstrated by the red-shifted extinction spec- tra as seen in Figure 6. Further, confocal microscopy successfully detected the bioconjugates on the HER2 positive cell line (Figure 8), indicating the success of the conjugation.

We believe that the mechanism of conjugation is the same as that in the case of gold nanospheres that is electrostatic and hydrophobic physisorption. It is also likely that at the $\mathrm{pH}$ at which the antibody is maintained, the Fc fragment of the antibody that is rich in positively charged amino acids such as lysine will bind to the negatively charged chloride ion layer on the exposed end faces of the rods. We intend to perform studies that will elucidate this aspect. Further, we will perform the protocol of first capping the nanorods with PSS for example, and then comparing the antigen binding affinity constants of the bioconjugates from the two methods.

\subsection{Potential contrast enhancing applications}

The scattering and absorption bands of the synthesized nanorods span the wavelength regime between $675-850 \mathrm{~nm}$ that is of interest to optical imaging. This occupies the most important part of the "optical imaging window" where light penetration in tissue is high due to reduced scattering and absorption coefficients. Optical imaging techniques (Table 2) that rely on scattering and/or absorption contrast to detect pathological tissue could benefit from the use of such nanoparticles with or without targeting capability. 
Our goal is to employ these particles as contrast agents for photoacoustic cancer imaging, which has been proposed earlier by Oraevsky and coworkers [26, 27]. Photoacoustic imaging relies on optical absorption for its signals. When photons are absorbed, nonradiative de-excitation of the absorbed optical energy takes place with the release of localized heat. The local thermal expansion that results produces pressure transients [5]. When illuminated with pulsed laser light, a tumor site by virtue of its higher absorption with respect to the healthy background tissue, due to angiogenesis [28], will act as a source of bipolar photoacoustic pulses. This ultrasound propagates with minimal distortion to the surface where it is detected using appropriate wideband detectors. The time-of-flight, amplitude, and peak-peak time of the bipolar PA pulse possess information regarding the location, absorption, and dimensions of the source, thereby permitting a reconstruction of the tumor site $[29,30]$.

It is known that the NIR optical absorption contrast of tumors versus healthy tissue, measured using optical mammographic methods, is between 1.5 and 3. Clinical trials of optical mammography are being conducted worldwide but at present, it seems implausible that intrinsic contrast alone will provide sufficient sensitivity and specificity, and targeted contrast enhancement is likely to be required [31]. Since the same contrast mechanism of optical absorption is operative in photoacoustic imaging as well, a similar conclusion may be anticipated.

An impression of the feasibility of using the nanorods synthesized for contrast enhancement is now discussed. The absorption cross-section of a nanorod at a wavelength, say $800 \mathrm{~nm}$, is estimated using discrete dipole approximation (DDSCAT) simulations $[32,33]$ as $C_{\mathrm{abs}}=2.8 \times 10^{-14} \mathrm{~m}^{2}$. A typical average optical absorption coefficient for an invasive ductal carcinoma is $\mu_{a}=0.008 \mathrm{~mm}^{-1}$ at $800 \mathrm{~nm}$. In order to achieve contrast enhancement, a certain number density of gold nanorods is required to exhibit higher absorption than the intrinsic value and may be calculated as

$$
\rho_{\mathrm{NR}} \geq \frac{\mu_{a}}{C_{\mathrm{abs}}} .
$$

This gives $\rho_{\mathrm{NR}}=2.8 \times 10^{8} \mathrm{NR} / \mathrm{cm}^{3}$. Further photoacoustic signals can be enhanced by a thermal nonlinearity mechanism to 3 orders of magnitude higher [34], then the modified number density of nanorods required is only $\rho_{\mathrm{NR}}=$ $2.8 \times 10^{5} \mathrm{NR} / \mathrm{cm}^{3}$.

Published studies report that most tumour cell types express from $2 \times 10^{4}$ to $20 \times 10^{4} \mathrm{ErbB} 2$ receptors/cell [12]. Let us assume arbitrarily that $2 \times 10^{3}$ of these sites per cell are occupied by conjugated nanorods. Further, if we assume that $1 \%$ of cells at a tumor site overexpress HER2 results in a figure of $2 \times 10^{6}$ cancer cells $/ \mathrm{cm}^{3}$. This will then lead to an estimation of the density of binding sites of the order of $10^{9} \mathrm{~cm}^{-3}$. Comparison of $\rho_{\mathrm{NR}}$ and the estimated figure of density of binding sites leads us to believe that contrast enhancement will be possible.

We intend to test these molecular probes in small animal photoacoustic imaging. Contrast enhancement with untargeted PEG-coated nanoparticles will be studied. Accumulation of the contrast agent at the tumor site will depend on enhanced permeation and retention (EPR). Active targeted studies will follow, with conjugated nanoparticles administered to the animal via the tail vein. In all studies, emphasis will be on ascertaining the sensitivity/efficacy of the technique with and without contrast agent.

\section{CONCLUSIONS}

We have synthesized gold nanorods with optical extinction peaks in the region from $675-850 \mathrm{~nm}$ making these eminently suited for scattering and absorption contrast enhancements in optical imaging. We have performed bioconjugation of these nanorods with HER81 antibodies, which bind with high efficiency to HER2 receptors expressed by SKBR3 breast carcinoma cells. We demonstrated in fixed cell studies that the targeting functionality of the antibody moiety remains viable. However, it must be mentioned that the situation in vivo will be complex compared to the simplified situation in vitro. Other unresolved issues remain at present. One of these is regarding toxicity and cellular uptake of these particles in vivo. Further, whether these molecular probes will be able to extravasate into the tumor tissue through leaks in the vasculature has not yet been studied. These are some lines of research that we intend to follow in the near future.

\section{ACKNOWLEDGMENTS}

We acknowledge fruitful discussions with Dr. Henk-Jan van Manen, Dr. Rolf Vermeij, and Dr. Christian Blum in various aspects related to bioconjugate chemistry, cell growth, and microscopy studies. The assistance of Sam Mathew (IIT Bombay) in early synthesis experiments is acknowledged. We thank Dr. Christina Graf (University of Wuerzburg) for discussions regarding the synthesis protocols. Peter van de Plas (Aurion, Wageningen, The Netherlands) is acknowledged for advice and tips on the use of bioconjugation protocols. We received the HER81 mAb and SKBR3 cells from Dr. Leon Terstappen and Dr. Arjan Tibbe (Immunicon). Electron Microscopy was carried out by Mark Smithers and Dr. Enrico Keim (CMAL/MESA ${ }^{+}$). We thank Frank Roesthuis (LT/TNW) for the use of fume hoods in the clean room. The research is funded by the University of Twente through the thrust area program NIMTIK, and by the Nederlandse Wetenschappelijk Organisatie (NWO) and Stichting Technische Wetenschappen (STW) through project TTF 6527. Simulations of optical properties of particles were performed using supercomputing facilities of the National Computing Facilities Foundation (NCF) supported by the NWO.

\section{REFERENCES}

[1] J. A. Conchello and J. W. Lichtman, "Optical sectioning microscopy," Nature Methods, vol. 2, no. 12, pp. 920-931, 2005.

[2] F. Helmchen and W. Denk, "Deep tissue two-photon microscopy," Nature Methods, vol. 2, no. 12, pp. 932-940, 2005.

[3] J. G. Fujimoto, "Optical coherence tomography for ultrahigh resolution in vivo imaging," Nature Biotechnology, vol. 21, no. 11, pp. 1361-1367, 2003. 
[4] D. A. Boas, D. H. Brooks, E. L. Miller, et al., "Imaging the body with diffuse optical tomography," IEEE Signal Processing Magazine, vol. 18, no. 6, pp. 57-75, 2001.

[5] M. Xu and L. V. Wang, "Photoacoustic imaging in biomedicine," Review of Scientific Instruments, vol. 77, no. 4, Article ID 041101, 22 pages, 2006.

[6] R. Richards-Kortum and E. Sevick-Muraca, "Quantitative optical spectroscopy for tissue diagnosis," Annual Review of Physical Chemistry, vol. 47, pp. 555-606, 1996.

[7] B. J. Tromberg, N. Shah, R. Lanning, et al., "Non-invasive in vivo characterization of breast tumours using photon migration spectroscopy," Neoplasia, vol. 2, no. 1-2, pp. 26-40, 2000.

[8] K. Licha, "Contrast agents for optical imaging," in Contrast Agents II: Optical, Ultrasound, X-Ray and Radiopharmaceutical Imaging, vol. 222 of Topics in Current Chemistry, chapter 2.1, pp. 1-29, Springer, Berlin, Germany, 2002.

[9] X. Intes, J. Ripoll, Y. Chen, S. Nioka, A. G. Yodh, and B. Chance, "In vivo continuous-wave optical breast imaging enhanced with indocyanine green," Medical Physics, vol. 30, no. 6, pp. 1039-1047, 2003.

[10] A. W. H. Lin, N. A. Lewinski, J. L. West, N. J. Halas, and R. A. Drezek, "Optically tunable nanoparticle contrast agents for early cancer detection: model-based analysis of gold nanoshells," Journal of Biomedical Optics, vol. 10, no. 6, Article ID 064035, 10 pages, 2005.

[11] J. Perez-Juste, I. Pastoriza-Santos, L. Liz-Marzan, and P. Mulvaney, "Gold Nanorods: Synthesis, Characterization and Applications," Coordination Chemistry Reviews, vol. 249, pp. 1870-1901, 2005.

[12] K. Sokolov, M. Follen, J. Aaron, et al., "Real-time vital optical imaging of precancer using anti-epidermal growth factor receptor antibodies conjugated to gold nanoparticles," Cancer Research, vol. 63, no. 9, pp. 1999-2004, 2003.

[13] D. A. Stuart, A. J. Haes, C. R. Yonzon, E. M. Hicks, and R. P. Van Duyne, "Biological applications of localised surface plasmonic phenomenae," IEE Proceedings Nanobiotechnology, vol. 152, no. 1, pp. 13-32, 2005.

[14] A. Gole and C. J. Murphy, "Seed-mediated synthesis of gold nanorods: role of the size and nature of the seed," Chemistry of Materials, vol. 16, no. 19, pp. 3633-3640, 2004.

[15] C. J. Murphy, T. K. Sau, A. M. Gole, et al., "Anisotropic metal nanoparticles: synthesis, assembly, and optical applications," Journal of Physical Chemistry B, vol. 109, no. 29, pp. 1385713870, 2005.

[16] N. R. Jana, L. Gearheart, and C. J. Murphy, "Seed-mediated growth approach for shape-controlled synthesis of spheroidal and rod-like gold nanoparticles using a surfactant template," Advanced Materials, vol. 13, no. 18, pp. 1389-1393, 2001.

[17] B. Nikoobakht and M. A. El-Sayed, "Preparation and growth mechanism of gold nanorods (NRs) using seed-mediated growth method," Chemistry of Materials, vol. 15, no. 10, pp. 1957-1962, 2003.

[18] T. K. Sau and C. J. Murphy, "Seeded high yield synthesis of short au nanorods in aqueous solution," Langmuir, vol. 20, no. 15, pp. 6414-6420, 2004.

[19] C. J. Orendorff and C. J. Murphy, "Quantitation of metal content in the silver-assisted growth of gold nanorods," Journal of Physical Chemistry B, vol. 110, no. 9, pp. 3990-3994, 2006.

[20] D. J. Slamon, G. M. Clark, S. G. Wong, W. J. Levin, A. Ullrich, and W. L. McGuire, "Human breast cancer: correlation of relapse and survival with amplification of the HER-2/neu oncogene," Science, vol. 235, no. 4785, pp. 177-182, 1987.

[21] D. A. Handley, "Methods for synthesis of colloidal gold," in Colloidal Gold: Principles, Methods, and Applications, pp. 1332, Academic Press, New York, NY, USA, 1989.

[22] B. Nikoobakht and M. A. El-Sayed, "Evidence for bilayer assembly of cationic surfactants on the surface of gold nanorods," Langmuir, vol. 17, no. 20, pp. 6368-6374, 2001.

[23] M. Liu and P. Guyot-Sionnest, "Mechanism of silver(I)assisted growth of gold nanorods and bipyramids," Journal of Physical Chemistry B, vol. 109, no. 47, pp. 22192-22200, 2005.

[24] G. T. Hermanson, "Preparation of colloidal-gold-labeled proteins," in Bioconjugate Techniques, pp. 593-605, Academic Press, New York, NY, USA, 1996.

[25] X. Huang, I. H. El-Sayed, and M. A. El-Sayed, "Cancer cell imaging and photothermal therapy in the near-infrared region by using gold nanorods," Journal of the American Chemical Society, vol. 128, no. 6, pp. 2115-2120, 2006.

[26] J. A. Copland, M. Eghtedari, V. L. Popov, et al., "Bioconjugated gold nanoparticles as a molecular based contrast agent: implications for imaging of deep tumors using optoacoustic tomography," Molecular Imaging and Biology, vol. 6, no. 5, pp. 341-349, 2004.

[27] M. Eghtedari, M. Motamedi, V. L. Popov, N. A. Kotov, and A. A. Oraevsky, "Optoacoustic imaging of gold nanoparticles targeted to breast cancer cells," in Photons Plus Ultrasound: Imaging and Sensing, vol. 5320 of Proceedings of the SPIE, pp. 21-28, San Jose, Calif, USA, January 2004.

[28] P. Carmeliet and R. K. Jain, "Angiogenesis in cancer and other diseases," Nature, vol. 407, no. 6801, pp. 249-257, 2000.

[29] S. Manohar, A. Kharine, J. C. G. van Hespen, W. Steenbergen, and T. G. van Leeuwen, "Photoacoustic mammography laboratory prototype: imaging of breast tissue phantoms," Journal of Biomedical Optics, vol. 9, no. 6, pp. 1172-1181, 2004.

[30] S. Manohar, A. Kharine, J. C. G. van Hespen, W. Steenbergen, and T. G. van Leeuwen, "The Twente Photoacoustic Mammoscope: system overview and performance," Physics in Medicine and Biology, vol. 50, no. 11, pp. 2543-2557, 2005.

[31] H. Rinneberg, D. Grosenick, K. T. Moesta, et al., "Scanning time-domain optical mammography: detection and characterization of breast tumors in vivo," Technology in Cancer Research and Treatment, vol. 4, no. 5, pp. 483-496, 2005.

[32] B. T. Draine and P. J. Flatau, "User Guide for the Discrete Dipole Approximation Code DDSCAT.6.1," http://arxiv.org/ abs/astro-ph/0409262.

[33] B. T. Draine and P. J. Flatau, "Discrete-dipole approximation for scattering calculations," Journal of the Optical Society of America, vol. 11, no. 4, pp. 1491-1499, 1994.

[34] I. G. Calasso, W. Craig, and G. J. Diebold, "Photoacoustic point source," Physical Review Letters, vol. 86, no. 16, pp. 35503553, 2001. 

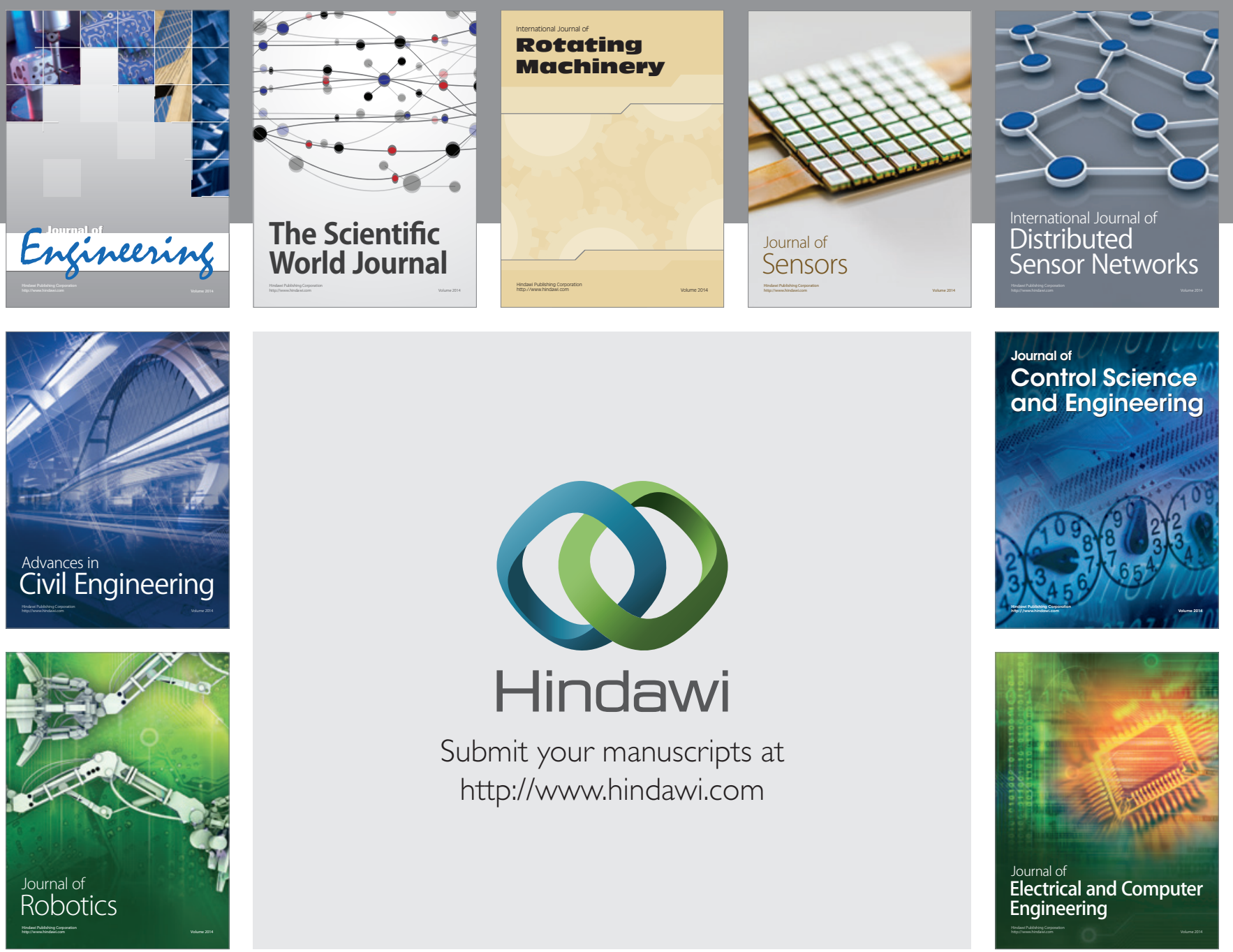

Submit your manuscripts at

http://www.hindawi.com
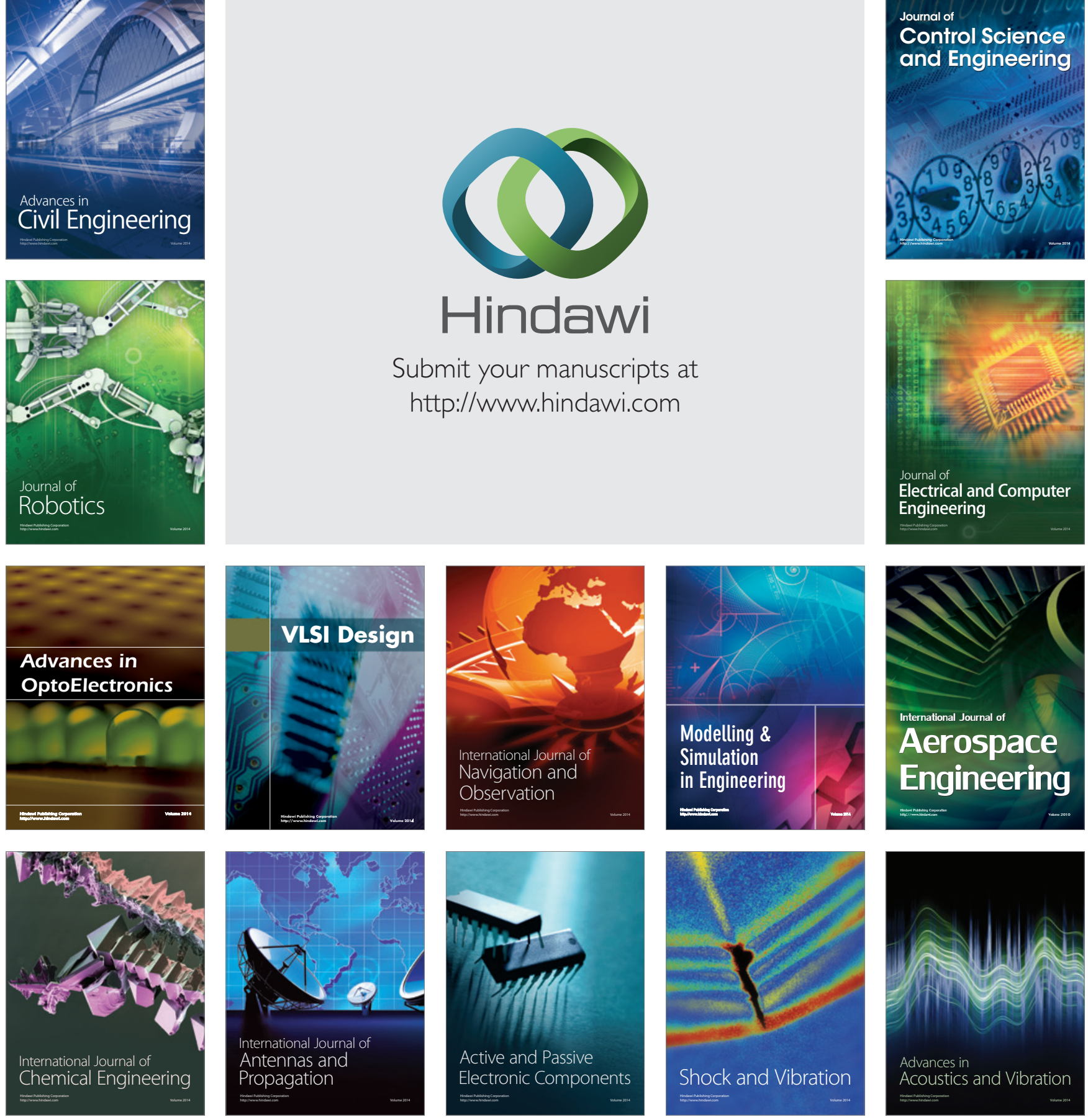